\title{
CONSUMO DE SUSTANCIAS PSICOACTIVAS: UN ESTUDIO DESCRIPTIVO-TRANSVERSAL EN LA UNIVERSIDAD DEL AtLÁNTICO
}

\section{Psychoactive Substance Use: A Descriptive Cross-Sectional Study at the University of Atlantico}

\author{
Dolores Nieto-Ortiz ${ }^{1}$, IsaAc Nieto-Mendoza ${ }^{2}$ \\ https://doi.org/10.17533/udea.rp.v12n1a01
}

\section{Resumen}

El objetivo de esta investigación es describir la presencia, la frecuencia, las circunstancias y los lugares de consumo de sustancias psicoactivas en los universitarios de la Licenciatura en Ciencias Sociales de la Universidad del Atlántico, como también sus actividades extraescolares. Su diseño es descriptivo-transversal, con una muestra no probabilística por conveniencia de 132 participantes. Se aplicó el cuestionario Percepción Social e Imaginarios Culturales de las Drogas en Población Universitaria (SIDEC). Los resultados encontrados evidencian que el alcohol es la sustancia psicoactiva de mayor consumo reciente $(43,9 \%)$, también la preva-

Recibido: 2020-04-23 / Aceptado: 2020-06-28

Para citar este artículo en APA: Nieto-Ortiz, D. y Nieto Mendoza, I. (2020). Consumo de sustancias psicoactivas: un estudio descriptivo-transversal en la Universidad del Atlántico. Revista de Psicología Universidad de Antioquia, 12(1), 7-24. doi: https:// doi.org/10.17533/udea.rp.v12n1a01 lencia de sustancias psicoactivas en el entorno universitario (principalmente tabaco, con un $100 \%$, y marihuana, con un $74,2 \%$ ); y preferencia por dedicar tiempo al ocio inactivo y consumo de sustancias psicoactivas en rumbas, bares y discotecas en circunstancias como fines de semana, viernes social y diversión $(51,5 \%)$, y espacios de la universidad (40,9\%), con el fin de relajarse, sentirse bien y por aburrimiento $(35,6 \%)$.

Palabras clave: sustancias psicoactivas, abuso de drogas, motivaciones para el consumo, prevalencia, estudiantes universitarios. 


\begin{abstract}
The objective of this research is to describe the presence, frequency, circumstances and places of consumption of psychoactive substances in the university students of the Bachelor of Social Sciences, University of Atlántico, as well as their extracurricular activities. A descriptive cross-sectional research design was used, with a non-probabilistic sample of 132 participants. The Social Perception and Cultural Imaginaries of Drugs in University Population (SIDEC) questionnaire was applied. The results found show that alcohol is the psychoactive substance with the highest recent consumption $(43.9 \%)$, as well as the
\end{abstract}

prevalence of psychoactive substances in the university environment (mostly tobacco, with $100 \%$, and marijuana, with $74.2 \%$ ); preference for dedicating time to inactive leisure and consumption of psychoactive substances in nightclubs, bars and discos in circumstances such as weekends, social Friday, and fun (51.5\%), and university spaces (40.9\%), also in order to relax, feel good and out of bore$\operatorname{dom}(35.6 \%)$.

Keywords: psychoactive substances, drug abuse, motivations for consumption, drug prevalence, university students.

\section{Introducción}

A nivel mundial, según los datos estadísticos de la Oficina de las Naciones Unidas para las Drogas y el Delito (UnOdC, 2018), "unos 275 millones de personas, es decir, el 5,6\% de la población mundial de edades comprendidas entre los 15 y 64 años, al menos alguna vez consumió drogas en el año 2016" (p. 1). Este informe resalta, entre los factores de mayor incidencia en el consumo, la depresión, la ansiedad, el trastorno de la conducta, el trastorno de déficit de atención con hiperactividad y el trastorno antisocial de la personalidad (unODC, 2018).

Diversas investigaciones realizadas en países latinoamericanos dan cuenta de la prevalencia del consumo de sustancias psicoactivas en universitarios, haciendo énfasis en la preferencia de sustancias legales, como el alcohol y el tabaco, e ilegales, como la marihuana y aquellas que se dan bajo prescripción médica; prevalencia que está asociada con el bullying y factores de riesgo como la disfunción familiar, el rechazo social, la baja autoestima, problemas académicos, la depresión, el estrés y el aislamiento social, entre otros (Alonso-Castillo et al., 2018; De la Torre et al., 2019; Mendoza-Carmona y Vargas-Peña, 2017; Urday-Concha et al., 2019). 
Respecto al consumo de sustancias psicoactivas en el contexto colombiano, según el último Estudio nacional de consumo de sustancias psicoactivas (2013), prima el consumo de alcohol (58,7\%), seguido por el tabaco $(16,2 \%)$. La marihuana $(3,3 \%)$ y la cocaína $(0,7 \%)$ cuentan con prevalencias más bajas (Ministerio de Salud y Protección Social, 2014). Específicamente en la categoría de sustancias ilícitas, según lo registra el Observatorio de Drogas de Colombia (oDC, 2017) de acuerdo con el testimonio de consumidores en edades de 12 a 65 años, el 87\% lo eran de marihuana, seguida por la cocaína, el bazuco y el éxtasis. Dichas cifras resultan preocupantes desde una perspectiva de salud pública, debido a la alerta de los entes gubernamentales colombianos sobre el crecimiento que se ha dado en los últimos años, especialmente en la edad escolar (oDC, 2017).

Además, según el boletín de prensa nro. 101 de 2018 del Ministerio de Salud y Protección Social, en Colombia hay preocupación en los entes gubernamentales por el incremento del consumo de marihuana, cocaína y, en general, demás sustancias psicoactivas en la población juvenil, debido al poco control por parte de las autoridades competentes en contextos como las universidades, donde se aprecia un mayor consumo, además de la falta de supervisión de los padres de familia, la disponibilidad de la sustancias psicoactivas, las condiciones socioeconómicas de los individuos, entre otros (Ministerio de Salud y Protección Social, 2018).

A nivel nacional, investigaciones en los departamentos de Antioquia, Santander, Pasto, Caldas, Tolima, Valle del Cauca y Risaralda (Betancourth et al., 2017; Castańo y Páez, 2019; Duarte et al., 2012; Mosquera et al., 2011; Páez et al., 2012; Restrepo et al., 2018; Soto et al., 2017) dan cuenta de cómo son las dinámicas con respecto al consumo de sustancias psicoactivas en universitarios, enfatizando que uno de los factores de riesgo determinantes es la disponibilidad de las sustancias en los entornos educativos, siendo notorio un alto consumo de alcohol, tabaco, marihuana y cocaína.

Particularmente en la ciudad de Barranquilla, de acuerdo con el oDC (2017), prevalece el consumo de marihuana, base de coca y fármacos obtenidos sin prescripción médica (Diazepam, Librium, Rohypnol o Rivotril); asimismo, en cuanto al consumo de drogas legales, como el alcohol y el tabaco, 
se encuentra entre las ciudades de mayor población con tendencia al alza de este tipo consumo. Investigaciones realizadas en esta ciudad reconocen el aumento en el consumo de alcohol y cigarrillo tanto en la universidad pública (Universidad del Atlántico) como en las privadas (Universidad Metropolitana, Universidad Simón Bolívar e Instituto Tecnológico de Soledad, Atlántico); también en el acceso a drogas ilegales, especialmente la marihuana y la cocaína (Candanoza et al., 2018; Caro, 2017; Cervantes et al., 2014; Nieto-Ortiz y Nieto-Mendoza, 2020).

Por otra parte, de acuerdo con la investigación realizada por Nieto-Ortiz y Nieto-Mendoza (2020), al analizar los estilos de vida de estudiantes de la Universidad del Atlántico, se identificó altos niveles de inactividad física, con una preferencia hacia prácticas poco saludables como el consumo excesivo de alcohol. Asimismo, el estudio realizado por Tirado et al. (2013) con universitarios españoles, identificó en estudiantes consumidores una menor preferencia por actividades deportivas y culturales y mayor interés en participar de actividades sociales, reuniones y fiestas. Otros estudios llevados a cabo en el departamento del Atlántico reconocen que el consumo de sustancias psicoactivas legales e ilegales en contextos universitarios públicos y privados se debe, en muchos casos, a la influencia de amistades en espacios extraescolares como las discotecas (Candanoza et al., 2018; Caro, 2017; Cervantes et al., 2014).

En línea con lo anterior, en esta investigación se propuso describir la presencia, la frecuencia, las circunstancias y los lugares de consumo de sustancias psicoactivas en los universitarios de la Licenciatura en Ciencias Sociales de la Universidad del Atlántico, así como sus actividades extraescolares.

\section{Metodología}

\section{Diseño}

La investigación se realizó bajo un diseño descriptivo-transversal. La muestra se seleccionó mediante un procedimiento no probabilístico por conveniencia (Hernández-Sampieri et al., 2014), de una población base de 742 universitarios. 
Consumo de sustancias psicoactivas: un estudio descriptivo-transversal en la Universidad del Atlántico

\section{Participantes}

La muestra estuvo constituida por 132 universitarios (69 hombres y $63 \mathrm{mu}-$ jeres) entre los 17 y 30 años de edad, matriculados en el semestre académico 2019-1 del programa Licenciatura en Ciencias Sociales de la Facultad de Educación de la Universidad del Atlántico. Se estableció como criterios de exclusión presentar antecedentes o alteraciones relacionadas con el consumo de sustancias psicoactivas o estar recibiendo algún tratamiento farmacológico o psicoterapéutico. Asimismo, la muestra fue seleccionada teniendo en cuenta la disponibilidad de la población, contactada a partir de los listados de cinco asignaturas específicas, brindados por la Facultad de Educación.

\section{Instrumento}

El instrumento utilizado fue el cuestionario Percepción Social e Imaginarios Culturales de las Drogas en Población Universitaria (SIDEC), validado por concepto de jueces, profesionales de la salud de Bienestar Universitario de la Universidad Tecnológica de Pereira (UTP) (Mosquera et al., 2011). Este formulario, de tipo autodiligenciado, aporta información complementaria respecto a los factores de análisis básicos como lo son la clase de sustancia consumida y la frecuencia, permitiendo realizar una caracterización sociodemográfica y de percepciones sociales e imaginarios culturales referentes al consumo de sustancias psicoactivas, fundamental para llevar a cabo investigaciones posteriores y la creación de estrategias de prevención.

Los indicadores que conforman la encuesta son los siguientes:

1- Disponibilidad de las sustancias psicoactivas: aquellas drogas que, según los estudiantes, pueden encontrarse en el contexto universitario.

2- Situación personal del consumo de las sustancias: esta se valora teniendo en cuenta la prevalencia de vida (proporción de estudiantes que consumieron alguna sustancia psicoactiva en algún momento de su vida), prevalencia del año (consumo de sustancias psicoactivas en los últimos 12 meses, denominado consumo reciente) y prevalencia del mes (estudiantes que consumieron algún tipo de sustancia en los últimos treinta días, denominado consumo actual durante el último mes o la última semana). 
3- Actividades extrauniversitarias: estas se clasificaron en ocupación de los estudiantes después de clases (actividades preferidas y horas semanales que les gustaría dedicar a ellas) y circunstancias y lugares asociados al consumo de sustancias psicoactivas, sea ocasional o habitualmente.

\section{Procedimiento}

Previo a la aplicación del instrumento, se procedió a la firma del consentimiento informado, seguido de un cuestionario de datos sociodemográficos para identificar estrato, semestre académico en curso y departamento de procedencia de los participantes. Posteriormente, se solicitó el diligenciamiento del cuestionario propuesto sobre consumo de sustancias psicoactivas.

\section{Consideraciones éticas}

Esta investigación fue aprobada el día 19 de febrero de 2019 por el Comité de Ética de la Dependencia de Investigaciones de la Universidad del Atlántico. Su desarrollo metodológico se adaptó a las disposiciones legales requeridas para estudios con humanos, establecidas en el Artículo 11 de la Resolución 8430 de 1993 del Ministerio de Salud y Protección Social, por el cual se clasificó este estudio como investigación sin riesgo. Asimismo, los estudiantes asintieron participar voluntariamente en la investigación por medio de la firma del consentimiento informado, en el cual se explicaron los fines de esta y las implicaciones de su participación, permitiéndoles retirarse en cualquier momento del desarrollo del estudio.

\section{Análisis estadístico}

Tras la obtención de los datos, estos fueron tabulados y procesados por medio del paquete estadístico IBM spss, versión 23. La interpretación de los datos se realizó mediante un análisis estadístico univariado, midiendo las variables en escala nominal y describiendo los datos mediante frecuencia y porcentaje. 
Consumo de sustancias psicoactivas: un estudio descriptivo-transversal en la

\section{Resultados}

\section{Caracteristicas sociodemográficas}

En la tabla 1 se presenta la caracterización sociodemográfica de la muestra estudiada, identificando que, en cuanto al sexo, estuvo conformada principalmente por hombres (52,3\%). En cuanto a la caracterización por edad, se evidenció una mayor frecuencia en el rango de 17 a 21 años $(67,4 \%)$ y menor en edades superiores a los 30 años (7,6\%). El promedio de edad de los estudiantes fue de 18,5 en hombres y 18,3 en mujeres. Con respecto al municipio de procedencia de los universitarios, el 90,2\% procedía del departamento del Atlántico y el 9,8\% del resto del país. Asimismo, los estudiantes de la Licenciatura en Ciencias Sociales encuestados se categorizaron por semestre académico en curso, hallándose mayor porcentaje en el rango de octavo a noveno semestre $(53,8 \%)$ y menor de primero a tercero $(22 \%)$.

Tabla 1

Caracterización sociodemográfica de la muestra estudiada

\begin{tabular}{llcc}
\hline \multirow{2}{*}{ Criterio } & & \multicolumn{2}{c}{ Valores } \\
\cline { 3 - 4 } & & $\mathrm{n}$ & $\%$ \\
\hline \multirow{2}{*}{ Sexo } & Hombres & 69 & 52,3 \\
& Mujeres & 63 & 47,7 \\
\hline \multirow{3}{*}{ Semestre } & $1-3$ & 29 & 22,0 \\
& $4-6$ & 32 & 24,2 \\
& $7-9$ & 71 & 53,8 \\
\hline \multirow{4}{*}{ Edad } & $17-21$ & 89 & 67,4 \\
& $22-26$ & 12 & 9,1 \\
& $27-30$ & 21 & 15,9 \\
\multirow{2}{*}{$\begin{array}{l}\text { Departamento de } \\
\text { procedencia }\end{array}$} & Mayores de 30 & 10 & 7,6 \\
\hline & Atlántico & 119 & 90,2 \\
& Resto del país & 13 & 9,8 \\
\hline
\end{tabular}


Sustancias que se pueden conseguir en la Universidad del Atlántico

Cuando se señalan los factores de riesgo del consumo de sustancias psicoactivas, es fundamental mencionar el acceso a estas. De acuerdo con los resultados expuestos en la tabla 2 , tanto drogas legales como ilegales pueden ser adquiridas dentro del contexto universitario. Según los encuestados, entre las sustancias psicoactivas legales, se puede obtener tabaco (100\%) y alcohol $(67,4 \%)$. Por su parte, entre aquellas sustancias psicoactivas ilegales y otras que deben ser consumidas solo bajo prescripción médica, se observa mayor prevalencia en la marihuana $(74,2 \%)$.

\section{Tabla 2}

Sustancias que consideran los estudiantes se pueden conseguir en la Universidad del Atlántico

\begin{tabular}{lcc}
\hline \multirow{2}{*}{ Sustancias } & \multicolumn{2}{c}{ Valores } \\
\cline { 2 - 3 } & $\mathrm{n}$ & $\%$ \\
\hline Tabaco & 132 & 100 \\
Marihuana & 98 & 74,2 \\
Alcohol & 89 & 67,4 \\
Éxtasis & 5 & 3,8 \\
Cocaína & 79 & 59,8 \\
Bazuco, base & 5 & 3,8 \\
Inhalables & 3 & 2,3 \\
Pepas/amitriptilina & 67 & 50,8 \\
Hongos & - & - \\
Heroína & - & - \\
Anfetaminas & - & - \\
Librium, Diazepam & 57 & 43,2 \\
LSD (ácido) & - & - \\
Rohypnol, Rivotril & 65 & - \\
Yagé & - & 49,2 \\
Prozac & - & - \\
\hline
\end{tabular}


Consumo de sustancias psicoactivas: un estudio descriptivo-transversal en la Universidad del Atlántico

\section{Prevalencia de consumo}

En relación con la prevalencia del consumo de sustancias psicoactivas en la Universidad del Atlántico, se obtuvieron los siguientes resultados, clasificados en prevalencia de vida, prevalencia del año y prevalencia del mes:

1- Prevalencia de consumo en la vida: en cuanto al porcentaje de universitarios que habían consumido alguna vez sustancias psicoactivas, se hizo palmaria la prevalencia del consumo de sustancias legales — tabaco y alcohol_- pues la totalidad de la muestra estudiada lo había hecho. Por su parte, con respecto a las sustancias ilegales y de acceso con prescripción médica, la marihuana fue la más consumida (94,7\%), seguida de Rohypnol/Rivotril (18,9\%), Pepas/amitriptilina (17,4\%), Librium/Diazepam (5,3\%) y yagé $(3,8 \%)$.

2- Prevalencia de consumo en el año: respecto al consumo de sustancias psicoactivas legales en los últimos 12 meses (figura 1), el 52,3\% de la muestra aseveró haber consumido alcohol, seguido del 34,8\% que consumió tabaco. Por su parte, en cuanto a las sustancias psicoactivas ilegales y de acceso con prescripción médica, prevaleció el consumo de marihuana (43,2\%), seguido de Rohypnol/Rivotril (12,9\%) Pepas/amitriptilina (9,8\%) y Librium/Diazepam (5,3\%).

Figura 1. Prevalencia anual de consumo en la Universidad del Atlántico

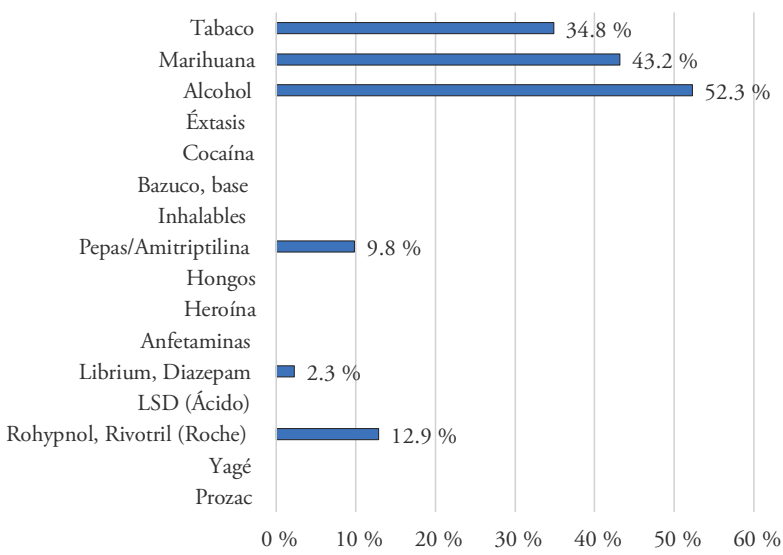


3- Prevalencia de consumo en el mes: referente al consumo actual de sustancias psicoactivas (últimos treinta días), se evidenció que, en cuanto a las sustancias legales, prevaleció el alcohol (43,9\%) sobre el tabaco $(29,5 \%)$. Por otro lado, la sustancia ilegal más consumida fue la marihuana $(31,8 \%)$, seguida de Rohypnol/Rivotril (7,6\%) y Pepas/amitriptilina (5,9\%).

\section{Actividades extrauniversitarias}

En torno a las actividades extraescolares, aquellas realizadas en el tiempo de ocio después de la jornada académica y las horas dedicadas a la misma (tabla 3), la totalidad de encuestados aseveró hacer uso del tiempo en actividades de carácter formativo-cognitivas y asociadas al uso de las tecnologías. Otros resultados relevantes tienen que ver con el uso del tiempo para trabajar y hacer diligencias (89,4\%), el ocio inactivo (ocio improductivo) (83,3\%) y las actividades de orden social-afectivo $(65,9 \%)$. De otro lado, las actividades asociadas al deporte contaron con un bajo porcentaje $(9,1 \%)$. Se hace palmario, además, el contraste del uso promedio de horas semanales en actividades asociadas al ocio inactivo (16 horas) respecto de las horas invertidas en actividades domésticas y el deporte (2 horas, respectivamente).

Ahora bien, en lo que concierne a las actividades que preferían los estudiantes y las horas a la semana que querían dedicarles (tabla 3), se presentó mayor interés en invertir tiempo en aquellas relacionadas con el uso de tecnologías (100\%), seguido del ocio inactivo $(83,3 \%)$, con una pretensión de emplear 16 horas a la semana a cada una de este tipo de actividades. Por el contrario, tan solo el 3,8\% tiene como interés practicar deporte y únicamente 2 horas a la semana. 
Consumo de sustancias psicoactivas: un estudio descriptivo-transversal en la

\section{Tabla 3}

Formas de emplear tiempo libre y actividades de preferencia por los universitarios

\begin{tabular}{|c|c|c|c|c|c|}
\hline & \multirow[b]{2}{*}{ Actividades } & \multicolumn{2}{|c|}{$\begin{array}{c}\text { Formas de emplear el } \\
\text { tiempo libre en horas } \\
\text { por semana }\end{array}$} & \multicolumn{2}{|c|}{$\begin{array}{l}\text { Actividades preferidas } \\
\text { de ocio y recreación }\end{array}$} \\
\hline & & $\begin{array}{c}\text { Promedio } \\
\text { de horas a la } \\
\text { semana }\end{array}$ & $\mathrm{n}(\%)$ & $\begin{array}{l}\text { Promedio } \\
\text { de horas a } \\
\text { la semana }\end{array}$ & n (\%) \\
\hline $\begin{array}{l}\text { Formativo- } \\
\text { cognitivo }\end{array}$ & $\begin{array}{l}\text { Estudiar, leer, escribir, asistir } \\
\text { a conferencias, investigar. }\end{array}$ & 14 & $132(100)$ & 10 & $21(15,9)$ \\
\hline Ocio inactivo & Dormir, ver TV, descanso. & 16 & $110(83,3)$ & 16 & $110(83,3)$ \\
\hline $\begin{array}{l}\text { Social, } \\
\text { afectivo }\end{array}$ & $\begin{array}{l}\text { Salir con amigos, rumbear, } \\
\text { visitar bares, discotecas, } \\
\text { tomar cerveza, salir a comer, } \\
\text { a tomar café, conversar, } \\
\text { compartir con novio(a), } \\
\text { tertuliar. }\end{array}$ & 11 & $87(65,9)$ & 11 & $58(43,9)$ \\
\hline Deporte & $\begin{array}{l}\text { Trotar, caminar, nadar, } \\
\text { montar bicicleta, ir al } \\
\text { gimnasio. }\end{array}$ & 2 & $12(9,1)$ & 2 & $5(3,8)$ \\
\hline $\begin{array}{l}\text { Cultural y } \\
\text { artístico }\end{array}$ & $\begin{array}{l}\text { Cine, arte en general, } \\
\text { escuchar música, tocar } \\
\text { guitarra o cualquier } \\
\text { instrumento musical, } \\
\text { dibujar. }\end{array}$ & 4 & $9(6,8)$ & 4 & $4(3)$ \\
\hline Tecnológico & $\begin{array}{l}\text { Usar el computador, } \\
\text { videojuegos, Internet, } \\
\text { chatear, hablar por celular. }\end{array}$ & 14 & $132(100)$ & 14 & $132(100)$ \\
\hline Familiar & $\begin{array}{l}\text { Compartir con los padres o } \\
\text { con la familia. }\end{array}$ & 6 & $7(5,3)$ & 4 & $2(1,5)$ \\
\hline $\begin{array}{l}\text { Turístico } \\
\text { ambiental }\end{array}$ & $\begin{array}{l}\text { Viajar, salir al campo, } \\
\text { acampar, etc. }\end{array}$ & 4 & $8(6,1)$ & 4 & $1(0,8)$ \\
\hline Ocupacional & Trabajar, hacer diligencias. & 12 & $118(89,4)$ & 6 & $17(12,9)$ \\
\hline Doméstico & $\begin{array}{l}\text { Hacer oficio, arreglar el } \\
\text { cuarto, etc. }\end{array}$ & 2 & $19(14,4)$ & 2 & $7(5,3)$ \\
\hline $\begin{array}{l}\text { Vocacional y } \\
\text { voluntariado }\end{array}$ & $\begin{array}{l}\text { Participar en organizaciones } \\
\text { juveniles, movimientos } \\
\text { políticos, Scouts, trabajo } \\
\text { comunitario. }\end{array}$ & - & - & - & - \\
\hline
\end{tabular}




\section{Circunstancias y lugares de consumo}

Finalmente, en la tabla 4 se presentan los resultados referentes a las circunstancias y lugares de consumo de sustancias psicoactivas. En cuanto a las circunstancias de consumo, el 35,6\% de la muestra aseveró consumir sustancias psicoactivas por relajarse, sentirse bien, desestresarse, por problemas personales, depresión, por aburrimiento, en momentos de tristeza, angustia y estrés. Respecto a los lugares de consumo, hubo mayor prevalencia en la respuesta que remite a rumbas, bares y discotecas en circunstancias como fines de semana, viernes social y diversión (51,5\%), seguido de universidad y cafeterías (40,9\%).

\section{Tabla 4}

Circunstancias y lugares asociados al consumo de sustancias psicoactivas

Circunstancias y lugares de consumo

\section{Circunstancias y lugares de consumo}

Rumbas, bares, discotecas, fines de semana, viernes social, diversión.

Conversando, reuniones con amigos, casas de amigos.

Fiestas de distinto tipo, celebraciones, reuniones sociales.

Celebraciones familiares, en casa, con parientes.

Paseos, fincas, campamentos, lugares especiales (ej.: campamento indígena).

Eventos especiales, conciertos, integraciones, todo tipo de actividades colectivas institucionales.

Por relajarse, sentirse bien, desestresarse, por problemas personales, depresión, por aburrimiento, en momentos de tristeza, angustia, estrés.

En la universidad, cafeterías.

Por probar.

Momentos de ocio.

Formulación médica.

De vez en cuando.

En cualquier momento y ocasión, cuando se desea.

$\begin{array}{cc}\text { Valores } \\ \mathrm{n} & \%\end{array}$

68 


\section{Discusión y conclusiones}

De acuerdo con los resultados obtenidos respecto a las posibilidades de acceso a sustancias psicoactivas en la Universidad del Atlántico, fue posible identificar, según los encuestados, que en la institución se logran obtener sustancias legales como tabaco (100\% de los reportes) y alcohol (67,4\% de los reportes); en lo concerniente a las sustancias ilegales: marihuana (74,2\% de los reportes), cocaína (59,8\% de los reportes), Pepas/amitriptilina (50,8\% de los reportes) y Rohypnol/Rivotril (Librium/Diazepam) (43,2\% de los reportes). Estos resultados están relacionados con los obtenidos por Mosquera et al. (2011), a partir de los cuales se identificó que en la Universidad Tecnológica de Pereira era posible tener acceso, dentro del contexto educativo, mayormente a la marihuana $(51,3 \%)$ y al tabaco $(67,2 \%)$.

Estos resultados también concuerdan con el estudio realizado en la Universidad de Santander (Bucaramanga) por Páez et al. (2012), en el que la posibilidad de acceso al alcohol fue reportada como elevada (83,7\%), distinto a la posibilidad de acceso a la de marihuana $(11,8 \%)$. Al contrastar los datos de la investigación con los obtenidos en el Estudio Nacional de Consumo de Sustancias Psicoactivas del Ministerio de Salud y Protección Social (2014), se evidencian discrepancias, especialmente en cuanto a la marihuana, siendo esta de menor acceso y consumo en Colombia (3,3\%).

Por otra parte, con respecto a la prevalencia de consumo en la vida, los universitarios manifestaron que el alcohol, el tabaco y la marihuana fueron probados alguna vez. Esto concuerda con los porcentajes obtenidos en la Universidad del Atlántico por Nieto-Ortiz y Nieto-Mendoza (2020), donde, de 404 estudiantes, el 76\% manifestó haber consumido alcohol; al igual que fue descrito en el estudio de Caro (2017), en el cual se evidenció que el alcohol fue la sustancia de mayor consumo $(57,43 \%)$ en una muestra de 418 estudiantes. Asimismo, los resultados del presente estudio, sobre este tipo de prevalencia, guardan relación con los obtenidos por Urday-Concha et al. (2019) al estudiar 86 universitarios e identificar la prevalencia del consumo de bebidas alcohólicas $(84,4 \%)$, aunque con respecto al consumo de marihuana $(17,1 \%)$ fuera notoriamente inferior, tal como en comparación con los resultados del estudio 
de Mosquera et al. (2011), puesto que solo el 16,7\% de los estudiantes aseveraron haber probado alguna vez la marihuana.

En la prevalencia de consumo en el último año, entre las drogas legales el consumo de alcohol obtuvo el mayor porcentaje (52,3\%); respecto a las ilegales, el consumo de marihuana (43,2\%). Estos resultados son menores a lo hallado en la investigación realizada por Betancourth et al. (2017) con 849 universitarios pastusos, identificando una frecuencia de consumo de alcohol en el último año de $97,5 \%$.

Al observar el consumo de sustancias psicoactivas en el último mes o consumo actual, el alcohol fue la sustancia mayormente consumida $(43,9 \%)$, y la droga ilegal de mayor prevalencia de consumo fue la marihuana $(31,8 \%)$, diferenciándose de la investigación realizada por Mosquera et al. (2011), puesto que en ella predominaron los inhalables $(6,2 \%)$, en lugar de la marihuana $(5,6 \%)$, en el último consumo. No obstante, la prevalencia de consumo de alcohol es menor a la de los resultados obtenidos en el estudio de Nieto-Ortiz y Nieto-Mendoza (2020), realizado en la Universidad del Atlántico, donde el consumo de alcohol fue del (76\%).

De las actividades extrauniversitarias llama la atención el uso de gran parte del tiempo de los universitarios en el ocio inactivo $(83,3 \%)$, en contraste con un bajo porcentaje en la práctica de deporte $(9,1 \%)$. Adicionalmente, los universitarios manifestaron querer pasar mayor tiempo haciendo uso de las tecnologías $(100 \%)$ y en el ocio inactivo $(83,3 \%)$. Estos resultados discrepan de los presentados en la investigación realizada por Mosquera et al. (2011), pues la mayoría de los universitarios preferían invertir tiempo en la práctica deportiva. En este sentido, los resultados se relacionan con los obtenidos por Caro (2017), debido a que en los universitarios que consumían alcohol, la prevalencia de inactividad física fue mayor, como fue notorio también en el estudio de Nieto-Ortiz y Nieto-Mendoza (2020).

Con respecto a las circunstancias y lugares de consumo, se destaca que los universitarios afirmaron consumir sustancias psicoactivas por relajarse, sentirse bien, desestresarse, por problemas personales, depresión, por aburrimiento, en momentos de angustia y estrés (35,6\%), indicando que el consumo lo pueden llegar a realizar en la universidad (40,9\%). Esto difiere del estudio 
realizado por Mosquera et al. (2011), en el cual el consumo de sustancias fue justificado principalmente por motivos de entretenimiento. En relación con estos datos, debe tenerse en cuenta que el Ministerio de Salud y Protección Social (2018) indica entre los factores de riesgo para el consumo de sustancias psicoactivas los problemas familiares, la baja tolerancia a la frustración, la baja autoestima y las situaciones de estrés. Por su parte, de acuerdo con el estudio de Cruz et al. (2018), las sustancias psicoactivas, tanto legales como ilegales, son consumidas con el fin de relajarse, desestresarse y dejar de pensar en situaciones familiares y otros problemas como las bajas calificaciones, la baja autoestima y el bullying.

A partir de los resultados obtenidos, se encuentra necesario que se planteen programas de prevención del consumo de sustancias psicoactivas dentro de la Universidad del Atlántico, induciendo a los estudiantes al aprovechamiento del tiempo de ocio de manera positiva, es decir, a la práctica de actividades deportivas, recreativas y lúdicas. Asimismo, deben realizarse campańas de sensibilización respecto a las implicaciones del consumo de sustancias psicoactivas y sus afectaciones en el desarrollo integral del individuo, como manifiestan Páez et al. (2012), señalando que, en programas de índole preventivo en materia de consumo de este tipo de sustancias, es preciso que se vincule al Departamento de bienestar universitario, a fin de brindar pautas direccionadas a la salud integral del estudiantado.

Por lo anterior, cabe señalar el aporte realizado por la investigación de Duarte et al. (2012), quienes consideran como estrategias de alto impacto para la prevención y disminución del consumo de sustancias psicoactivas en los contextos universitarios, aquellas intervenciones direccionadas al fortalecimiento y desarrollo de habilidades para la vida, relacionadas con la toma de decisiones, la solución de problemas y la asertividad, como también al uso del tiempo de ocio en actividades que beneficien la salud y promuevan estilos de vida saludable (Tirado et al., 2013; Nieto-Ortiz y Torrenegra, 2020).

Finalmente, los hallazgos de este estudio significan un aporte fundamental para el afrontamiento y creación de programas de intervención por parte del Departamento de Bienestar Universitario de la Universidad del Atlántico; no obstante, debido a las problemáticas que enfrentó la Universidad en el 
semestre académico 2019-1, debido al desarrollo interrumpido de las actividades académicas, no fue posible estudiar a un mayor número de estudiantes matriculados en el programa Licenciatura en Ciencias Sociales, lo que hubiese podido representar mayores conocimientos sobre las dinámicas referentes al consumo de sustancias psicoactivas y la prevalencia de las mismas dentro del contexto universitario. Aun así, se espera contribuir a programas institucionales que busquen atender a la población universitaria en relación con el abuso de sustancias psicoactivas, permitiendo la formulación de estrategias para que los estudiantes se apropien de conductas que denoten estilos de vida saludable.

\section{Referencias}

Alonso-Castillo, M., Armendaríz, N., López, K., Guzmán, F., y Esparza, S. (2018). Valores, estrés de conciencia y el consumo de alcohol, tabaco y drogas médicas en enfermería. Journal Health NPEPS, 3(1), 88-102. Recuperado de https:// periodicos.unemat.br/index.php/jhnpeps/article/view/2921

Betancourth, S., Tacán, L., y Córdoba, E. (2017). Consumo de alcohol en estudiantes universitarios colombianos. Universidad y Salud, 19(1), 37-50. doi: http:// dx.doi.org/10.22267/rus.171901.67

Candanoza, A., Consuegra, S., Rodríguez, P., Samper, J., y Manjarrés, E. (2018). Identificación y análisis de factores relacionados con el consumo de sustancias psicoactivas en la Institución Universitaria ITSA. Revista Colombiana Salud Libre, 13(2). doi: https://doi.org/10.18041/1900-7841/rcslibre.2018v13n2.4988

Caro, A. (2017). Factores individuales y socio-ambientales relacionados a la actividad física en universitarios de una institución de Barranquilla en 2017. Tesis de Maestría. Universidad del Norte, Barranquilla. Recuperado de http://manglar. uninorte.edu.co/bitstream/handle/10584/7886/131062.pdf?sequence=1

Castańo, J., y Páez, M. (2019). Funcionalidad familiar y tendencias adictivas a internet y a sustancias psicoactivas en estudiantes universitarios. Psicología desde el Caribe, 36(2), 117-206. Recuperado de http://rcientificas.uninorte.edu.co/ index.php/psicologia/article/viewFile/10090/214421444248

Cervantes, M., Acosta, J., Balaguera, K., Valenzuela, A., y Torres, A. (2014). Uso de alcohol en los estudiantes de tercero a quinto semestre del programa de Psicología de una universidad privada de la ciudad de Barranquilla. Psicogente, 17(32), 460-476. doi: https://doi.org/10.17081/psico.17.32.23 
Consumo de sustancias psicoactivas: un estudio descriptivo-transversal en la Universidad del Atlántico

Cruz, S., León, B., y Angulo, E. (2018). Lo que hay que saber sobre drogas. México D.F.: Río Arronte. Recuperado de https://www.researchgate.net/publication/328652667_Lo_que_hay_que_saber_sobre_drogas_2018_WEB

Duarte, C., Varela, M., Salazar, I., Lema, L., y Tamayo, J. (2012). Motivaciones y recursos para el consumo de sustancias psicoactivas en universitarios. Hacia la promoción de la salud, 17(1), 92-104. Recuperado de https://www.redalyc.org/ pdf/3091/309124894009.pdf

Hernández-Sampieri, R., Fernández, C., y Baptista, P. (2014). Metodología de la investigación (6. ${ }^{\text {ta }}$ ed.). México D.F.: McGraw Hill. Recuperado de http://observatorio.epacartagena.gov.co/wp-content/uploads/2017/08/metodologia-de-la-investigacion-sexta-edicion.compressed.pdf

Mendoza-Carmona, R., y Vargas-Peña, R. (2017). Factores psicosociales asociados al consumo y adicción a sustancias psicoactivas. Revista Electrónica de Psicología IZTACALA, 20(1), 139-167. Recuperado de https://www.medigraphic.com/ pdfs/epsicologia/epi-2017/epi171h.pdf

Ministerio de Salud y Protección Social. (Junio de 2014). Estudio Nacional de Consumo de Sustancias Psicoactivas en Colombia-2013. Informe final. Bogotá, Colombia. Recuperado de https://www.unodc.org/documents/colombia/2014/ Julio/Estudio_de_Consumo_UNODC.pdf

Ministerio de Salud y Protección Social. (24 de Agosto de 2018). Consumo de drogas es un tema de salud mental. Bogotá, Colombia. Recuperado de https://www. minsalud.gov.co/Paginas/Consumo-de-drogas-es-un-tema-de-salud-mental. aspx

Mosquera, J., Artamónova, I., y Mosquera, V. (2011). Consumo de sustancias psicoactivas en la Universidad Tecnológica de Pereira, Colombia. Investigaciones Andina, 13(22), 194-210. doi: https://doi.org/10.33132/01248146.258

Nieto-Ortiz, D., y Nieto-Mendoza, I. (2020). Estilo de vida de estudiantes de la Universidad del Atlántico: un estudio descriptivo-transversal. Ciencia e Innovación en Salud, 1-16. doi: https://doi.org/10.17081/innosa.69

Nieto-Ortiz, D., y Torrenegra-Ariza, S. (2019). Relación entre el índice de masa corporal y actividad física en universitarios de la ciudad de Barranquilla en el semestre 2018-2, Universidad del Atlántico. Revista Biociencias, 14(1). doi: https://doi.org/10.18041/2390-0512/biociencias.1.5441

Observatorio de Drogas de Colombia. (Octubre de 2017). Reporte de drogas de Colombia-2017. Bogotá. Recuperado de http:/www.odc.gov.co/Portals/1/publicaciones/pdf/odc-libro-blanco/reporte_drogas_colombia_2017.pdf 
Páez, A., Solano, S., Duran, M., Suárez, E., Melgarejo, P., Flórez, G., y Ortiz, E. (2012). Prevalencia de consumo de sustancias psicoactivas en estudiantes universitarios de la salud y ciencias sociales. Revsta Cuidarte, 3(1), 334-341. Recuperado de https://www.redalyc.org/pdf/3595/359533179011.pdf

Restrepo, J., Amador, O., Calderón, G., Castañeda, T., Osorio, Y., y Diez, A. (2018). Depresión y su relación con el consumo de sustancias psicoactivas, el estrés académico y la ideación suicida en estudiantes universitarios colombianos. Health and Adictions, 18(2), 227-239. doi: http://dx.doi.org/10.21134/haaj.v18i2.359

Soto, M., Bastidas, C., y Quimbayo, J. (2017). Consumo de sustancias psicoactivas en estudiantes universitarios en el área de la salud. Salud, Historia y Sanidad, 12(2), 29-48. Recuperado de http://fredmanrique.com/ojs/index.php/SHS/ article/view/181/179

Tirado, R., Aguaded, J., y Marín, I. (2013). Incidencia de los hábitos de ocio y consumo de drogas sobre las medidas de prevención de la drogadicción en universitarios. Educación XXI, 16(2), 137-160. Recuperado de https://www.redalyc.org/ pdf/706/70626451008.pdf

Torre de la, R., Intriago, S., Zambrano, C., y Cedeño, Z. (2019). El consumo de sustancias psicotrópicas de los estudiantes del Cantón Bolívar, Provincia de Manabí, en Ecuador. Revista Digital Cientifica, 2(15), 1-13. Recuperado de https:// www.itsup.edu.ec/myjournal/index.php/sinapsis/article/view/227/260

UNODC (2018). Resumen, conclusiones y consecuencias en materia de políticas. Informe mundial sobre drogas. Ginebra. Recuperado de https://www.unodc.org/ wdr2018/prelaunch/WDR18_ExSum_Spanish.pdf

Urday-Concha, F., González, C., Peñalva, L., Pantigoso, E., Cruz, C., y Pinto, L. (2019). Percepción de riesgos y consumo de drogas en estudiantes universitarios de enfermería, Arequipa, Perú. Enfermería Anual en Costa Rica, (36), 1-5. doi: http://dx.doi.org/10.15517/revenf.v0i36.3341 